REVIEW

\title{
Outcomes of inpatient mobilization: a literature review
}

\author{
Beatrice J Kalisch, Soohee Lee and Beverly W Dabney
}

\begin{abstract}
Aims and objectives. To review current research evidence on the outcomes of mobilising hospitalised adults.
Background. Although immobility is known to cause functional decline or complications, inpatient ambulation emerged as the most often missed element of nursing care. This study is designed to review research studies that give evidence as to the consequences of mobilising or not mobilising hospitalised adult patients.

Design. A literature review of published peer-reviewed empirical research was undertaken.

Methods. The electronic databases of MEDLINE (Ovid), CINAHL, and PubMed were accessed to search for relevant empirical articles, supplemented by a search of reference lists contained in retrieved articles and citation tracking.

Results. Thirty-six studies were identified for inclusion in the review. Four areas (study design, sample size, measurement and statistical analysis) were evaluated for methodological quality, and most studies showed strong quality. A synthesis of the findings generated four themes of the effects of inpatient mobilisation: (1) physical outcomes included pain, deep vein thrombosis, fatigue, etc.; (2) psychological outcomes included anxiety, depressive mood, distress, comfort and satisfaction; (3) social outcomes included quality of life and independence; and (4) organisational outcomes included length of stay, mortality and cost.

Conclusion. Mobilising hospitalised adults brings benefits for not only physical functioning, but also their emotional and social well-being. Moreover, ambulation yields important organisational benefits. These benefits of mobilisation on four areas required viewing the patient in a holistic manner. Even though each study approached different types of patients, illnesses and procedures, this review showed that most inpatients would benefit from mobilisation and would experience optimal functions.

Relevance to clinical practice. The importance of mobilisation for positive patient outcomes highlights the need to develop methods to ensure that this nursing action is completed on a systematic basis.
\end{abstract}

Key words: ambulation, early ambulation, early mobilisation, hospitalisation, inpatients, mobilisation, mobility

Accepted for publication: 25 January 2013

\section{Introduction}

In several studies of missed nursing care, defined as required nursing care that is omitted or significantly delayed, ambulation of patients was identified as the most frequently missed element of inpatient nursing care, missed $76 \cdot 1-88.7 \%$ of the time (Kalisch et al. 2009a,b, 2011). Callen et al. (2004) also found inpatient ambulation to be a missed component of nursing care during an observational study of hallway ambulation on three medical units where $19 \%$ of patients walked once, $5 \%$ walked twice, $3 \%$ walked more than twice and $73 \%$ did not walk at all during the study period. Brown et al. (2004) also uncovered inadequacies in inpatient mobilisation. Observation of 45 hospitalised medical patients indicated that, on average, $83 \%$ of the hospital stay was spent lying in bed. The amount of time spent standing or walked ranged from $0 \cdot 2-21 \%$.
Authors: Beatrice J Kalisch, PhD, RN, FAAN, Director, Innovation and Evaluation and Titus Professor, School of Nursing, University of Michigan, Ann Arbor, MI; Soohee Lee, BSN, MSN, RN, Doctoral Student, School of Nursing, University of Michigan, Ann Arbor, MI; Beverly W Dabney, PhD, RN, Associate Professor, Southwestern Adventist University, Keene, TX, USA
Correspondence: Soohee Lee, Doctoral Student, School of Nursing, University of Michigan, 1115 Maiden Lane Court, Apt. 104, Ann Arbor, MI 48105, USA. Telephone: +1 7348466519 .

E-mail: soohee@umich.edu 
The fact that ambulation of hospitalised adults is regularly missed indicates that many patients are confined to bed or a chair and are mostly immobile throughout their hospital stay. Studies exploring inpatient immobility have uncovered several negative consequences of bed rest effecting the cardiovascular, respiratory, gastrointestinal, integumentary, musculoskeletal, renal, endocrine and nervous systems (Creditor 1993, Convertino 1997, Graf 2006). Patients begin to experience a decline in walking ability within two days of being hospitalised (Hirsch et al. 1990). Lack of inpatient mobility can be especially devastating to the older where the ageing process contributes to more rapid functional decline (Graf 2006). This new walking dependence among the older population may lead to discharge to a nursing home and has been found to result in continued walking dependence three months after discharge in $27 \%$ of older patients (Mahoney et al. 1998).

To understand the impact of mobilising patients in acute care hospitals, we reviewed the research literature to uncover and synthesise the relevant research evidence.

\section{Aims}

The aim of this paper was to provide a review of the literature related to the outcomes of mobilising (or not mobilising) adult patients in acute care settings. A review of current literature was conducted to identify relevant articles on inpatient mobilisation outcomes.

\section{Methods}

For this review, mobilisation was defined as walking, standing or sitting in a chair with or without assistance. The terms of early ambulation and early mobilisation were included in the definition of mobilisation. Early ambulation and early mobilisation refer to specific situations and are dependent upon the type of surgery, procedure or illness. For example, for cardiac catheterisation patients, early mobilisation was considered three to four hours after the procedure (Chair et al. 2007). For total knee replacement patients, early mobilisation was considered 24 hours after the surgery (Pearse et al. 2007). In addition, for stroke patients, early mobilisation was considered to be within 24-36 hours after symptom onset (Langhorne et al. 2010, Cumming et al. 2011).

\section{Search strategy}

The key terms used in the literature search included ('inpatients' or 'hospitalization' or 'hospitalized patients') AND ('ambulation' or 'early ambulation') OR ('mobilization' or 'early mobilization') OR ('mobility'). The electronic databases of MEDLINE (Ovid), CINAHL and PubMed were accessed. This process was supplemented by a search of reference lists contained in retrieved articles and citation tracking.

\section{Study criteria}

We reviewed published studies that met the following inclusion criteria: (1) empirical research that included a report of outcomes related to inpatient mobilisation, (2) published in peer-reviewed journals between 1999-2011 (to retrieve the most up-to-date evidence), (3) written in English and (4) whose population consisted of adult inpatients in acute care hospital settings.

Studies were excluded if they (1) took place in nonacute healthcare settings such as outpatient clinics, nursing homes, patient homes, etc.; (2) took place in an inpatient rehabilitation unit, psychiatric unit or the emergency department; (3) included a paediatric population; (4) included other types of mobilisation such as range of motion, turning or specialised mobilisation including aerobic exercises, bicycling or weight training.

\section{Article selection and analysis}

Potentially relevant studies included 462 records identified in CINAHL, 614 articles found in MEDLINE and 9452 records identified in PubMed. After duplicates were removed, 10,528 titles and abstracts were screened for relevance to inpatient mobilisation by the authors. This resulted in an initial selection of 171 articles. These 171 studies were entered into the full-text review stage and were independently analysed by the authors to determine whether they met the inclusion criteria. Of these, 148 studies did not meet the inclusion criteria and were eliminated. A total of 13 additional studies were added from hand searching of reference lists and citation tracking. After review of the full text and detailed evaluation by the three authors, 36 studies were selected for inclusion in this review (Fig. 1). Articles passing the full-text screening were placed in a data extraction form, and a list of included studies was created. To manage bias, all 36 studies were reviewed independently by the three authors. The reported outcomes of inpatient mobilisation that were aggregated into a data extraction form were categorised into themes. A summary of findings under the emergent themes is provided below.

\section{Quality of studies}

Thirty-six studies were evaluated by the three authors for methodological quality relative to study design, sample size, 


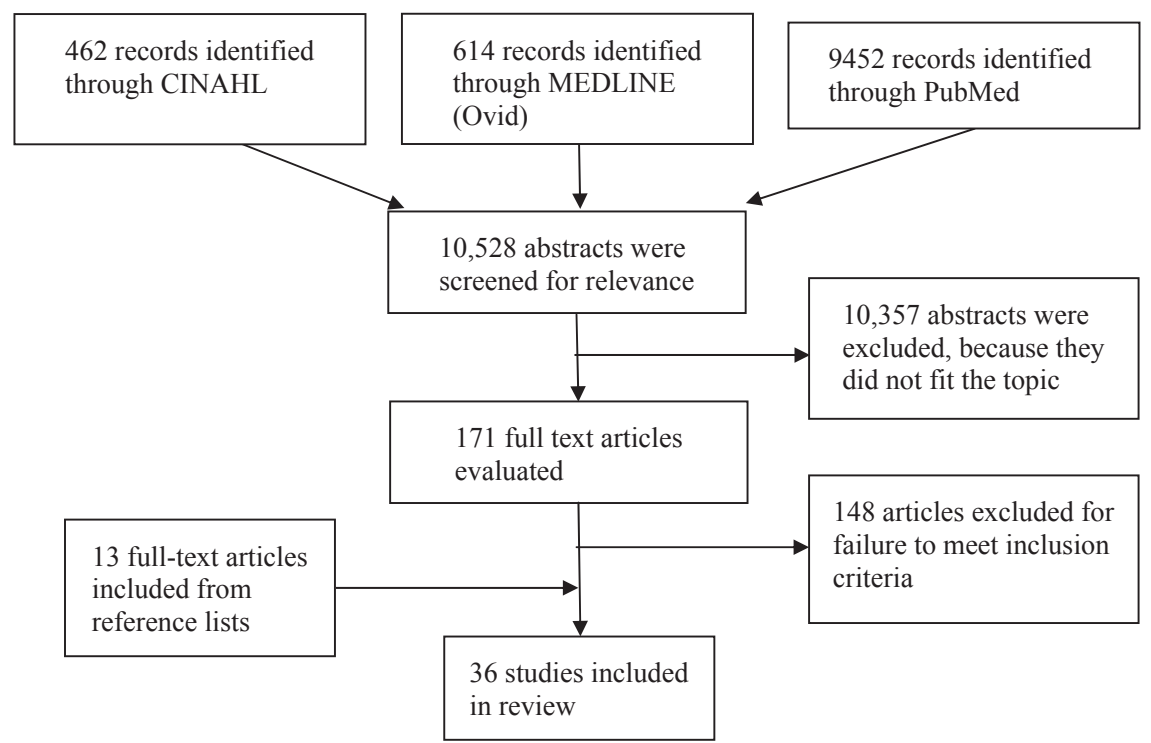

Figure 1 A flowchart of the search methods used in determining the articles used in this review. measurement and statistical analysis. Criteria to guide scoring these four areas were developed by the three authors based on published criteria and their research experience (Estrabrooks 2003, Wong \& Cummings 2007, De Cordova et al. 2012). A total of 12 possible points could be assigned. Study design was scored as 3 (randomised controlled trial), 2 (quasi-experimental study) or 1 (observational study). Sample size was scored as 3 (adequate sample sizes based on appropriate calculations), 2 (small sample size) or 1 (pilot study). Measurement was scored as 3 (adequate reliability and validity of measures), 2 (mixed reliability and validity of measures) or 1(no reliability and validity available). Statistical analysis was scored as 3 (completely adequate analysis), 2 (partially adequate analysis) or 1 (inadequate analysis).

\section{Results}

\section{Quality of studies}

Of the 36 studies evaluated, quality scores ranged from 7-11. Studies with scores of 1-4 were considered weak studies, 5-8 moderate, and 9 or higher strong. Of these, 27 studies received scores of 9-11 which we evaluated to be strong and nine studies showed moderate quality with scores of 7-8. None of the studies were categorised as weak. The lowerlevel studies shared nonexperimental designs or small sample sizes that limited generalisability of the findings; however, their results were promising and consistent with other studies and contributed to the findings of this review. Of the 36 studies, 27 studies tested interventions, of which 26 studies included control groups in their designs (Table 1).

\section{Search results}

Table 2 presents the summary of studies included in this review. The main outcomes were classified into four categories: physical outcomes, psychological outcomes, social outcomes and organisational outcomes (Table 3).

\section{Physical outcomes}

There were 21 studies that suggested physical benefits of hospital mobilisation, including pain relief (Partsch \& Blattler 2000, Chair et al. 2007, Augustin et al. 2010), less deep vein thrombosis (DVT; Pearse et al. 2007, Chandrasekaran et al. 2009, García Guerrero et al. 2010, Langhorne et al. 2010, Nakao et al. 2010), less fatigue (Chang et al. 2008, Rezaei-Adaryani et al. 2009), incidence of new pneumonia (Kamel et al. 2003, Kurabe et al. 2010), less delirium (Kamel et al. 2003, Schweickert et al. 2009), more ventilator-free days (Schweickert et al. 2009), less urinary tract infection (Kurabe et al. 2010, Langhorne et al. 2010) and improved physical function (Killey \& Watt 2006, Oldmeadow et al. 2006, Chang et al. 2008, Hirschhorn et al. 2008, Padula et al. 2009, Langhorne et al. 2010, Cumming et al. 2011, Zisberg et al. 2011).

Pain relief was found as the most frequently observed positive outcome of inpatient mobilisation. Partsch and Blattler (2000) conducted a randomised controlled trial of patients in the acute stage of DVT. Patients receiving compression stockings and ambulation had a significant reduction in pain after the second day as well as less swelling, compared to those on bed rest and no compression 
Table 1 Summary of quality assessment

\begin{tabular}{|c|c|c|c|c|c|}
\hline Author, date & Study design & Sample size & $\begin{array}{l}\text { Measurement } \\
\text { (reliability and validity) }\end{array}$ & Statistical analysis & Quality score \\
\hline Augustin et al. (2010) & Quasi-experimental & 347 & Adequate & Completely adequate & 10 \\
\hline Behnke et al. (2003) & Quasi-experimental & 26 & Mixed & Completely adequate & 9 \\
\hline Brown et al. (2004) & Prospective observational & 498 & Mixed & Completely adequate & 9 \\
\hline Browning et al. (2007) & Prospective observational & 50 & Mixed & Completely adequate & 8 \\
\hline Chandrasekaran et al. (2009) & Quasi-experimental & 100 & Mixed & Inadequate & 8 \\
\hline Chang et al. (2008) & Quasi-experimental & 22 & Mixed & Completely adequate & 8 \\
\hline Chair et al. (2007) & Quasi-experimental & 86 & Mixed & Completely adequate & 10 \\
\hline Craig et al. (2010) & Meta-synthesis & 103 & Mixed & Partially adequate & 10 \\
\hline Cumming et al. (2008) & Quasi-experimental & 71 & Mixed & Completely adequate & 10 \\
\hline Cumming et al. (2011) & Quasi-experimental & 71 & Mixed & Completely adequate & 10 \\
\hline Delaney et al. (2003) & Quasi-experimental & 64 & Mixed & Completely adequate & 10 \\
\hline Fisher et al. (2011) & Retrospective case control & 10 & Mixed & Completely adequate & 7 \\
\hline Fisher et al. (2010) & Prospective cohort & 162 & Mixed & Completely adequate & 9 \\
\hline Frenea et al. (2004) & Quasi-experimental & 61 & Mixed & Completely adequate & 10 \\
\hline García Guerrero et al. (2010) & Quasi-experimental & 47 & Mixed & Inadequate & 7 \\
\hline Hirschhorn et al. (2008) & Quasi-experimental & 93 & Mixed & Completely adequate & 10 \\
\hline Indredavik et al. (1999) & Quasi-experimental & 220 & Mixed & Completely adequate & 10 \\
\hline Kamel et al. (2003) & Retrospective cohort & 131 & Mixed & Completely adequate & 9 \\
\hline Killey and Watt (2006) & Quasi-experimental & 55 & Mixed & Completely adequate & 9 \\
\hline Kurabe et al. (2010) & Quasi-experimental & 182 & Mixed & Completely adequate & 10 \\
\hline Langhorne et al. (2010) & Quasi-experimental & 32 & Mixed & Completely adequate & 8 \\
\hline Larsen et al. (2009) & Quasi-experimental & 87 & Mixed & Partially adequate & 9 \\
\hline Mundy et al. (2003) & Quasi-experimental & 458 & Mixed & Completely adequate & 10 \\
\hline Nakao et al. (2010) & Comparative & 37 & Mixed & Completely adequate & 8 \\
\hline Oldmeadow et al. (2006) & Quasi-experimental & 60 & Adequate & Completely adequate & 11 \\
\hline Padula et al. (2009) & Quasi-experimental & 50 & Adequate & Partially adequate & 9 \\
\hline Partsch and Blattler (2000) & Quasi-experimental & 45 & Mixed & Completely adequate & 9 \\
\hline Pearse et al. (2007) & Quasi-experimental & 195 & Mixed & Completely adequate & 10 \\
\hline Rath et al. (2010) & Quasi-experimental & 23 & Mixed & & 8 \\
\hline Rezaei-Adaryani et al.(2009) & Quasi-experimental & 70 & Mixed & Completely adequate & 10 \\
\hline Schweickert et al.(2009) & Quasi-experimental & 104 & Mixed & Completely adequate & 10 \\
\hline Shadmi and Zisberg (2011) & Prospective cohort & 485 & Mixed & Partially adequate & 8 \\
\hline Siu et al. (2006) & Prospective cohort & 532 & Mixed & Completely adequate & 9 \\
\hline Tay-Teo et al. (2008) & Quasi-experimental & 71 & Mixed & Partially adequate & 9 \\
\hline Tyedin et al. (2010) & Quasi-experimental & 71 & Mixed & Completely adequate & 11 \\
\hline Zisberg et al. (2011) & Prospective observational & 525 & Mixed & Completely adequate & 9 \\
\hline
\end{tabular}

stockings. Pain relief with ambulation has also been found in posttransfemoral cardiac catheterisation patients. Patients in the experimental group were ambulated four hours after bed rest, while the control group was mobilised at the usual care time of 12-24 hours postcardiac catheterisation. The experimental group experienced less back pain and less urinary discomfort than the control group (Chair et al. 2007). Augustin et al. (2010) studied postpercutaneous coronary intervention patients and found less pain in the intervention group which ambulated three hours after the procedure than in the control group which ambulated 10 hours after the procedure.

Deep vein thrombosis prevention was also a prevalent outcome of inpatient mobilisation. Chandrasekaran et al.
(2009) explored early mobilisation of total knee replacement patients who began sitting out of bed or walking on the first postoperative day. Compared to the control group, the early mobilisation group had significantly less incidence of thromboembolic complications. Pearse et al. (2007) found that initiating walking within 24 hours of knee replacement surgery significantly decreased the incidence of DVT. Nakao et al. (2010) discovered that in patients with osteoarthritis or rheumatoid arthritis, ambulating early after total knee arthroplasty surgery significantly lowered D-dimer levels. García Guerrero et al. (2010) studied 47 consecutive patients receiving temporary pacemaker implantation and found that those who had a high or moderate amount of mobility did not experience a DVT, and 


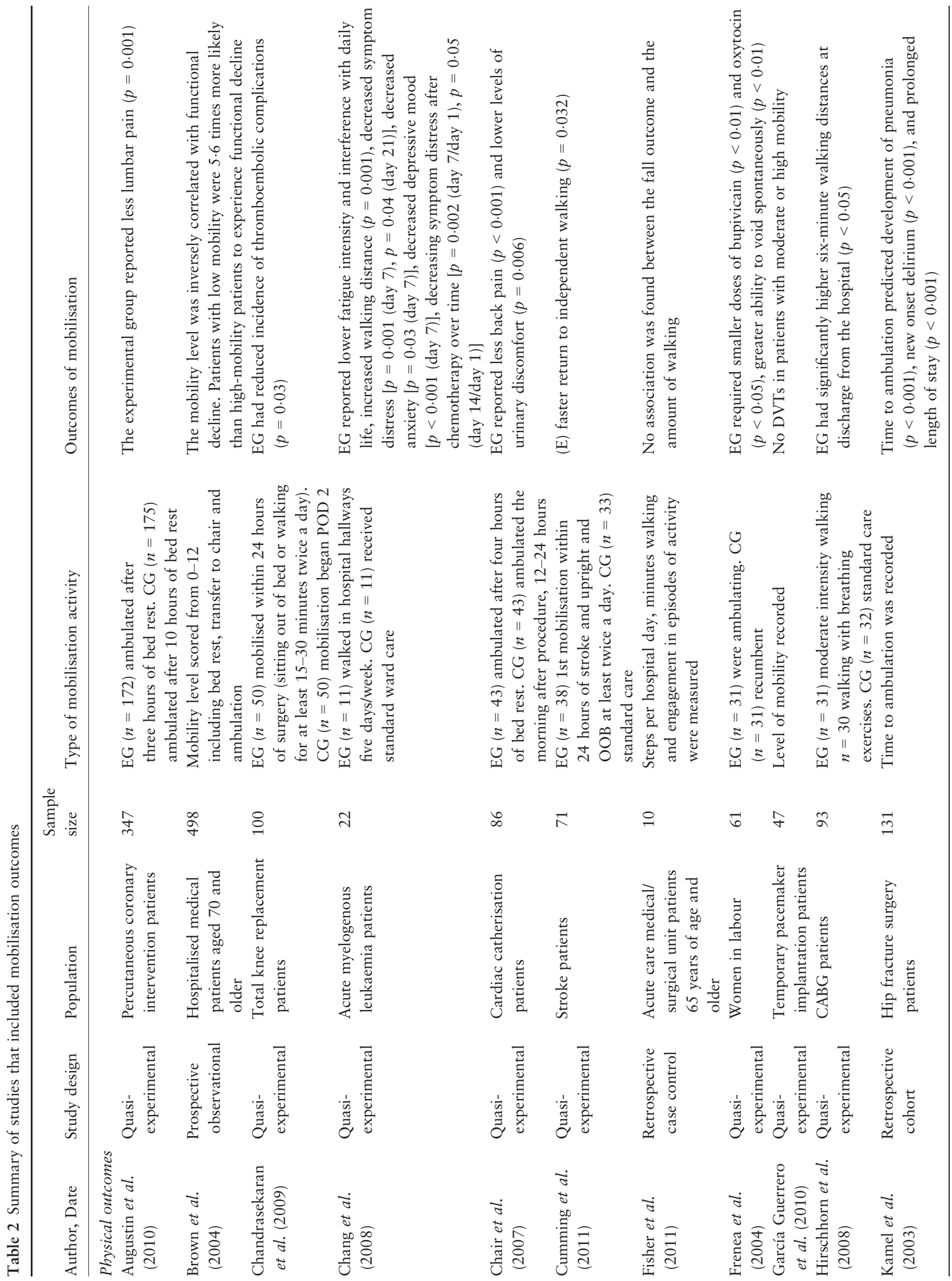




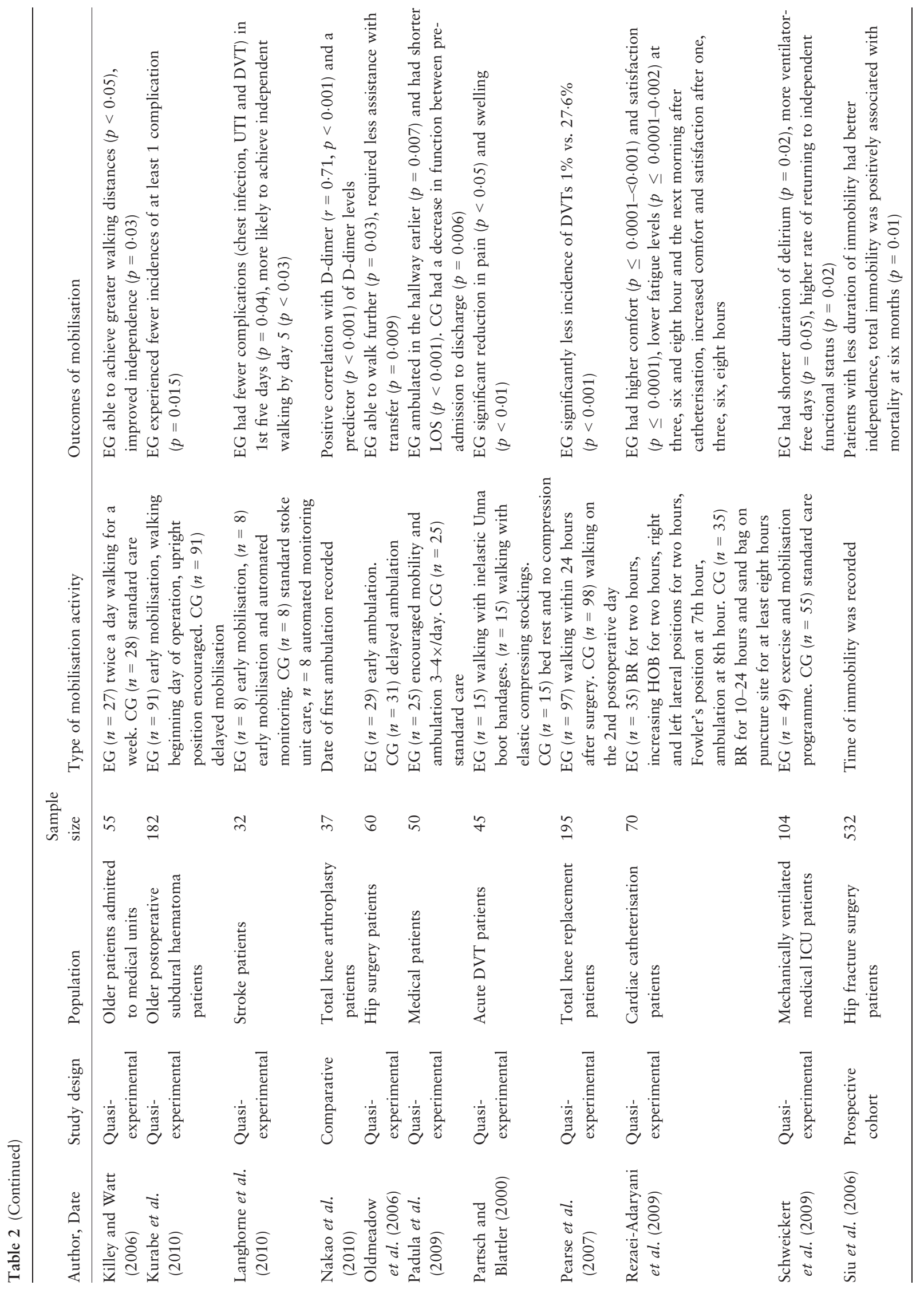




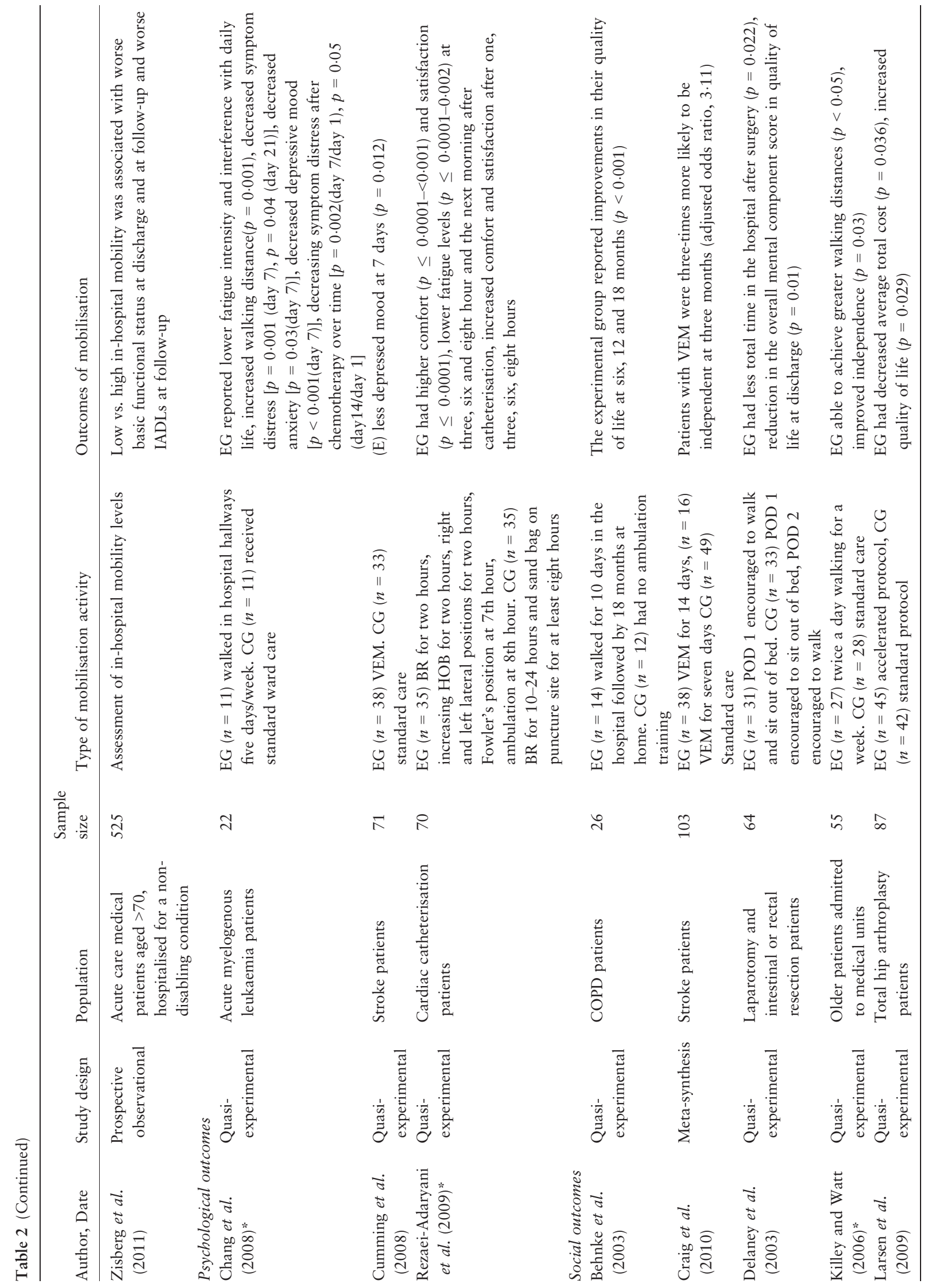




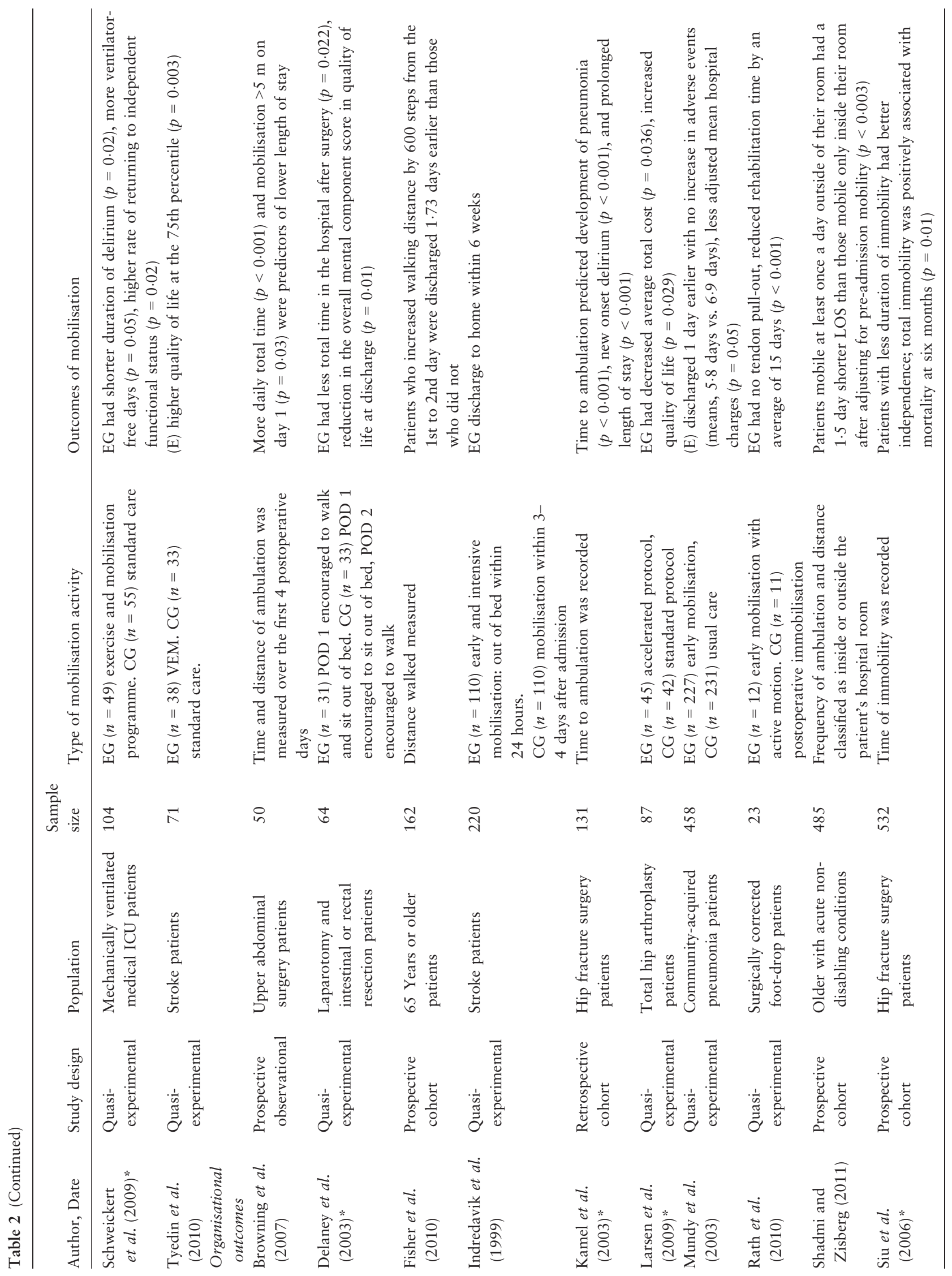




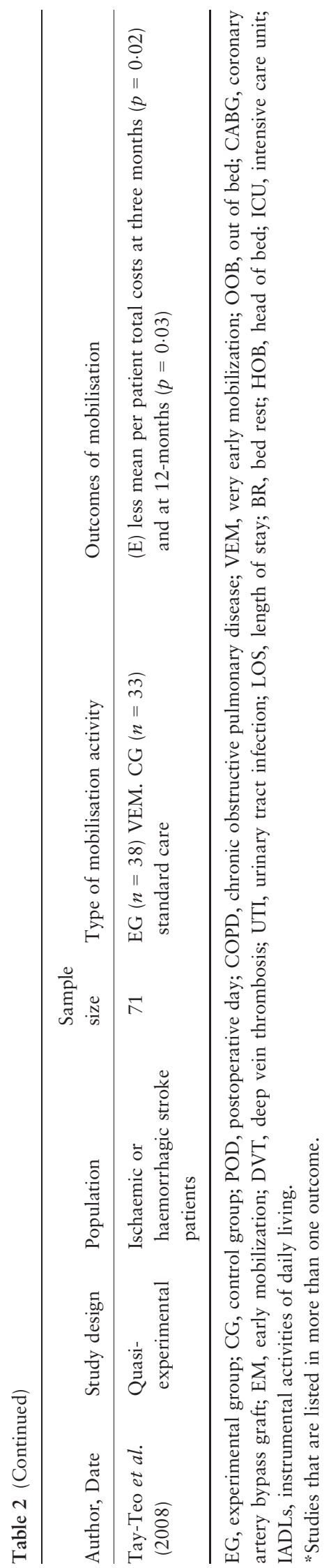

$6.4 \%$ of patients with a low amount of mobility did develop a DVT.

Fatigue was another outcome studied. Chang et al. (2008) explored the effects of walking on fatigue-related experiences of acute myelogenous leukaemia (AML) patients. Patients were randomised into an experimental group, which received a three-week walking exercise programme or a control group experiencing standard care. The walking group had lower levels of fatigue intensity and interference with daily life. The effects of position change and earlier ambulation on patient fatigue and comfort were examined in cardiac catheterisation patients (Rezaei-Adaryani et al. 2009). In this study, the experimental group received intermittent position change for the first six hours after cauterisation and ambulated seven hours after the procedure. The control group received routine care and was restricted to bed rest for 10-24 hours in a supine position. The experimental group reported less fatigue.

The review revealed additional effects of mobilisation on physical outcomes such as reduced incidence of new pneumonia, delirium, urinary tract infection and more ventilator-free days. Kamel et al. (2003) found that the longer the time to ambulation after hip fracture surgery, the greater the chance for development of pneumonia and new onset delirium. Schweickert et al. (2009) also evaluated effects of early exercise and mobilisation on critically ill intensive care unit mechanically ventilated patients and found that the intervention group experienced shorter duration of delirium and more ventilator-free days. Kurabe et al. (2010) evaluated the number of complications in older chronic subdural haematoma patients who underwent one burr-hole surgery. The early mobilisation group, who began walking on the day of the surgery, experienced fewer postoperative complications of pneumonia and urinary tract infections than the control group. Langhorne et al. (2010) found that in stroke patients, the early mobilisation group was less likely to develop complications of immobility such as chest infection, urinary tract infection and a DVT in the first five days.

Several studies showed improvement in physical function after inpatient mobilisation. Three studies exploring the impact of hospital ambulation on walking capacity indicated a positive effect (Killey \& Watt 2006, Chang et al. 2008, Hirschhorn et al. 2008). Killey and Watt (2006) examined the impact of providing hospitalised older adults two extra walks per day. Compared to the control group, the intervention group demonstrated increased mobility measured by their ability to walk further distances. A study of hospitalised AML patients found that a three-week walking exercise programme increased 12-minute walking 
Table 3 Main categories and outcomes of impatient mobilisation

\begin{tabular}{|c|c|c|}
\hline Categories & Outcomes & \\
\hline \multirow[t]{13}{*}{ Physical outcomes } & Less delirium & Kamel et al. (2003), Schweickert et al. (2009) \\
\hline & Less pain & Partsch and Blattler (2000), Chair et al. (2007), Augustin et al. (2010) \\
\hline & $\begin{array}{l}\text { No relationship with } \\
\text { inpatient falls }\end{array}$ & Fisher et al. (2011) \\
\hline & Less urinary discomfort & Chair et al. (2007) \\
\hline & Improved ability to void & Frenea et al. (2004), Augustin et al. (2010) \\
\hline & Less urinary tract infection & Kurabe et al. (2010), Langhorne et al. (2010) \\
\hline & Less fatigue & Chang et al. (2008), Rezaei-Adaryani et al. (2009) \\
\hline & Less DVT & $\begin{array}{l}\text { Pearse et al. (2007), Chandrasekaran et al. (2009), García Guerrero et al. (2010), } \\
\text { Langhorne et al. (2010), Nakao et al. (2010) }\end{array}$ \\
\hline & Less pneumonia & Kamel et al. (2003), Kurabe et al. (2010) \\
\hline & More ventilator-free days & Schweickert et al. (2009) \\
\hline & Increased walking distance & Killey and Watt (2006), Chang et al. (2008), Hirschhorn et al. (2008) \\
\hline & $\begin{array}{l}\text { Faster return to independent } \\
\text { ambulation }\end{array}$ & Siu et al. (2006), Langhorne et al. (2010), Cumming et al. (2011) \\
\hline & Improved physical function & $\begin{array}{l}\text { Killey and Watt (2006), Oldmeadow et al. (2006), Chang et al. (2008), } \\
\text { Hirschhorn et al. (2008), Padula et al. (2009), Langhorne et al. (2010), } \\
\text { Cumming et al. (2011), Zisberg et al. (2011) }\end{array}$ \\
\hline \multirow{5}{*}{$\begin{array}{l}\text { Psychological } \\
\text { outcomes }\end{array}$} & Less depression & Chang et al. (2008), Cumming et al. (2008) \\
\hline & Less anxiety & Chang et al. (2008) \\
\hline & Increased comfort & Rezaei-Adaryani et al. (2009) \\
\hline & More satisfaction & Rezaei-Adaryani et al. (2009) \\
\hline & Less symptom distress & Chang et al. (2008) \\
\hline \multirow[t]{2}{*}{ Social outcomes } & Improved quality of life & Behnke et al. (2003), Delaney et al. (2003), Larsen et al. (2009), Tyedin et al. (2010) \\
\hline & More independence & Killey and Watt (2006), Schweickert et al. (2009), Craig et al. (2010) \\
\hline \multirow[t]{3}{*}{$\begin{array}{l}\text { Organisational } \\
\text { outcomes }\end{array}$} & Decreased length of stay & $\begin{array}{l}\text { Indredavik et al. (1999), Kamel et al. (2003), Delaney et al. (2003), } \\
\text { Mundy et al. (2003), Browning et al. (2007), Fisher et al. (2010), Rath et al. (2010), } \\
\text { Shadmi and Zisberg (2011) }\end{array}$ \\
\hline & Less mortality & Mundy et al. (2003), Siu et al. (2006), Tay-Teo et al. (2008) \\
\hline & Less cost & Mundy et al. (2003), Tay-Teo et al. (2008), Larsen et al. (2009) \\
\hline
\end{tabular}

DVT, deep vein thrombosis.

distance ability compared to standard inpatient care (Chang et al. 2008). Hirschhorn et al. (2008) studied coronary artery bypass graft patients. They found that patients in the walking and walking/breathing groups had significantly higher six-minute walking distances than the 'gentle mobilisation' group upon discharge from the hospital. Oldmeadow et al. (2006) studied the function of 60 hip surgery patients after early ambulation. Patients were randomised to either an early ambulation group, which began walking postoperative day 1 or 2 , or a delayed ambulation group, which began walking postoperative day 3 or 4 . They found that the early ambulation group had significantly better functional recovery at postoperative day 7 and were able to walk on average twice as far as the delayed ambulation group and required less assistance to transfer and ambulate. Padula et al. (2009) studied the effects of a nurse-driven mobility protocol on the functional status of inpatients. They found that the treatment group ambulated in the hallway earlier [2.7 days vs. 4.9 days $(p=0.007)]$ and the control group had a statistically significant decrease in function between preadmission and discharge $(p=0.006)$. Zisberg et al. (2011) examined the mobility levels of 525 older adults and the association between mobility levels and functional outcomes. They found that low vs. high mobility to be associated with poorer basic functional status at discharge and also at follow-up. The low-mobility patients also had poorer instrumental activities of daily living at follow-up.

Inpatient ambulation has also been found to help patients' return to independent walking. Cumming et al. (2011) explored the use of early and more intense mobilisation on stroke patients. They found that patients who had their first mobilisation within 24 hours of the stroke and were out of bed at least twice a day returned to walking unassisted sooner than the standard care group. Langhorne et al. (2010) also studied stroke patients and found that early mobilisation patients were more likely to achieve walking by day 5 of their hospital admission and were less likely to develop complications of immobility. Examining the 
association between the length of immobility and function in hip fracture patients found that those who were gotten out of bed earlier had better function at two months (Siu et al. 2006). However, no significant differences were found at six months, indicating an initial benefit of faster recovery.

A few studies found negative or inconclusive effects of mobilisation on physical outcomes. Augustin and colleagues (2010) revealed no significant improvement in the frequency of urinary retention among percutaneous coronary intervention patients in the early ambulation group, with urinary retention being the same in both groups. Using the pain visual analogue scale scores or the length of labour, Frenea et al. (2004) investigated the effects of ambulating women in labour on the duration of labour and pain and found no significant difference between the ambulation and the recumbent group. They did however find that the ambulatory group required smaller doses of bupivicain and oxytocin and had a greater ability to void spontaneously. Fisher et al. (2011) studied acute care patients aged 65 and older to explore the effects of inpatient mobilisation on falls. Examination of total patient steps per day, minutes walking and engagement of activity did not reveal an association between increased mobility and inpatient falls.

\section{Psychological outcomes}

Three articles suggested an effect of mobilisation on psychological outcomes such as anxiety and depressive mood (Chang et al. 2008, Cumming et al. 2008), symptom distress (Chang et al. 2008), and comfort and satisfaction (Rezaei-Adaryani et al. 2009).

Mobilisation had positive effects on anxiety, depressive mood and symptom distress. Chang et al. (2008) studied the effects of walking exercise programme on cancer patients and found improvement in mood and symptom distress. Even though the effect of walking on anxiety and depressive mood diminished over time, this study supports the positive psychological effect of ambulation on cancer patients undergoing chemotherapy. In addition, the results of the control group indicate that patients experience emotional distress during chemotherapy, especially during the initial period, emphasising the need of an intervention to decrease their emotional distress. Cumming et al. (2008) explored the effects of very early mobilisation (VEM) on depression and anxiety of stroke patients and found that the VEM group showed a less depressed mood at seven days after a stroke compared to the group with standard care, which entailed less ambulation. However, the VEM group had only marginally less anxiety than the control group, not strongly supporting the effect of ambulation on anxiety.
Comfort and satisfaction were studied as other psychological outcomes of patient mobilisation. Rezaei-Adaryani et al. (2009) investigated the effect of early ambulation after cardiac catheterisation on comfort and satisfaction of patients. The levels of comfort and satisfaction until the next morning after catheterisation increased in the experimental group but decreased in the control group. These differences between the two groups support the positive effect of early ambulation on patients' comfort and satisfaction.

\section{Social outcomes}

Five articles examined how ambulation helps patients in living after discharge by studying quality of life (Behnke et al. 2003, Delaney et al. 2003, Larsen et al. 2009, Tyedin et al. 2010) and independence (Killey \& Watt 2006, Schweickert et al. 2009, Craig et al. 2010).

Inpatient mobilisation was found to positively influence the quality of life of patients. Tyedin et al. (2010) emphasised that patients tend to have a lower quality of life after stroke and suggested early mobilisation to recover it. The study found that the mobilisation group had significantly improved independent physical function, one subdomain of quality of life, as compared to the control group. Behnke et al. (2003) also supported the improvement in quality of life for patients with chronic obstructive pulmonary disease. In the study by Delaney et al. (2003), the mental component system, one subcategory of quality of life, resulted in improvement only at the time of discharge, but not at tenor 30 days after discharge. Larsen et al. (2009) identified that among total hip arthroplasty patients, the accelerated group had additional average gain in health-related quality of life compared to those who received the standard protocol.

Independence was another social outcome of inpatient mobilisation. Craig et al. (2010) conducted a meta-analysis of two trials: a very early rehabilitation trial and a very early rehabilitation contrasted with intensive telemetry after stroke (VERITAS). From individual data, it was concluded that stroke patients with early ambulation were three times more likely to be independent at three months. Exercise and mobilisation also have a positive effect on independence for critically ill patients. Schweickert et al. (2009) evaluated the performing activities of daily living at hospital discharge and found that patients under exercise intervention had a higher rate of returning to independent functional status. Killey and Watt (2006) applied extra walking to the intervention group in the medical unit and found that the level of independence in the walking group increased from admission day to after seven days while it decreased in the control group. The result showed the 
significant role of a walking programme on improved independence of older patients.

\section{Organisational outcomes}

The effects of inpatient mobilisation on hospital organisational outcomes included issues such as length of stay at the hospital (Indredavik et al. 1999, Delaney et al. 2003, Kamel et al. 2003, Mundy et al. 2003, Browning et al. 2007, Fisher et al. 2010, Rath et al. 2010, Shadmi \& Zisberg 2011), mortality (Mundy et al. 2003, Siu et al. 2006, Tay-Teo et al. 2008) and cost (Mundy et al. 2003, Tay-Teo et al. 2008, Larsen et al. 2009).

Mundy et al. (2003) applied early mobilisation on patients with community-acquired pneumonia and showed that patients receiving early mobilisation discharged one day earlier than the control group with no increase in adverse events. Delaney et al. (2003) compared two different types of postoperative care for patients after laparotomy and intestinal resection. One pathway was traditional care and other was controlled rehabilitation with early ambulation and diet (CREAD). Even though CREAD is a multimodal approach, early ambulation formed a greater part of the intervention. The result demonstrated a reduction in hospital stay in the CREAD group. Padula et al. (2009) also found in their study of a nurse-driven mobility protocol that patients in the treatment group had significantly shorter length of stays (4.96 days vs. 8.75 days, $p<0.001$ ). Rath et al. (2010) compared mobilisation to immobilisation, which is a conventional management for patients after a tendon transfer. The early mobilisation group was applied a splint instead of a cast which was removed five days after surgery while the immobilised group was applied a cast which was removed 29 days after the procedure. Following the rehabilitation protocol for three weeks after removing the splint or cast, the patients in the mobilised group were discharged 15 days earlier than the immobilised group with no tendon insertion pull-out, the major complication of the surgery. This study supported the advantage of early ambulation after foot-drop correction.

Several researchers found that the amount of time of ambulation predicted or influenced length of stay. Fisher et al. (2010) calculated total steps of geriatric patients to measure ambulation and indicated that low or negative step change score from the first to second day were associated with longer lengths of stay. Browning et al. (2007) also investigated the quantity of ambulation and found that mobilisation $>5 \mathrm{~m}$ on the first day and the amount of time of being upright were predictors of length of stay along with duration of anaesthesia and intensive care admission. Shadmi and Zisberg (2011) studied hospitalised adults aged
70 years and older with acute nondisabling conditions and found that those who ambulated outside of their room at least once a day had a 1.5 day shorter length of stay than those who only ambulated inside of their room. These results remained significant after adjusting for pre-admission mobility levels. Moreover, Indredavik et al. (1999) identified which aspects of the stroke unit contributed to the improved result in treatment. There were several important characteristics of stroke unit care, but shorter time to start systematic mobilisation was the most critical factor associated with 'discharge to home within six weeks'.

Regarding the issue of mortality, there were inconsistent findings. Siu et al. (2006) examined the effect of immobility of patients after hip fracture surgery on mortality at six months after discharge and showed that six-month survival was worse with delays in getting patients out of bed. However, Mundy et al. (2003) and Tay-Teo et al. (2008) did not find any significant difference in mortality rates between the early mobilisation group and control group.

Finally, the cost and efficiency outcomes of inpatient mobilisation were studied as outcomes of mobilisation. TayTeo et al. (2008) found that the VEM group incurred significantly less costs (determined from medical records and patient interviews) at three and 12 months and less demand of rehabilitation services. The cost difference at three months was largely attributable $(84 \%)$ to lower inpatient rehabilitation costs among VEM patients. Cost was saved only at 12 months in the mobilisation group when productive loss was added, but it can be concluded that cost savings were attributable to the lesser amount of inpatient rehabilitation. Larsen et al. (2009) estimated average total cost, postoperative productivity loss and hospitalisation cost (preoperative and perioperative) and identified that among total hip arthroplasty patients, the accelerated group was less costly compared to those who received the standard protocol. In addition, Mundy et al. (2003) estimated $\$ 1000$ per patient was saved under early mobilisation, showing the effect of ambulation on hospital charges.

\section{Discussion}

Findings from the literature review demonstrated various benefits of mobilising hospitalised adults. The majority of studies focused on the physical outcomes of inpatient mobilisation, followed by organisational, social and psychological outcomes, respectively. The physical benefits of inpatient mobilisation included less delirium, pain, urinary discomfort, urinary tract infection, fatigue, DVT, pneumonia, more ventilator-dependent days and improved ability to void. The physical function benefits also included 
improved walking distance and shorten time to return of independent ambulation. This result provides insight into the impact of mobilisation for hospitalised adults: patients who experience less mobilisation and prolonged immobility often experience less optimal physical and psychosocial outcomes, slower recovery, more functional decline and longer length of stays, than patients with more mobility. The type of mobilisation activity and the timing of this mobilisation may vary dependent on patient characteristics, illness and procedures. However, based on the results of this review, most inpatients would benefit from inpatient mobilisation and would experience less than optimal outcomes if this activity is omitted.

There was evidence in this review that mobilisation affects not only patients' physical functioning, but also their emotional and social well-being. Mobilisation decreased depression, anxiety and symptom distress and enhanced more comfort and satisfaction. It also enhanced quality of life and independence. This review requires viewing the patient in a holistic manner. In addition to benefits to patients, organisational benefits were uncovered including cost reduction, decreased length of stays and lower mortality rates. This finding indicates that inpatient mobility is not only good for the patients, but also the organisations that care for them. Even though a few studies demonstrated negative or inconclusive findings, most studies found positive effects of inpatient mobilisation and emphasised the importance of mobilisation.

\section{Limitations}

Assessment of the quality of all studies found two weaknesses: (1) varied sample sizes which ranged from 22-458 in experimental design studies and from 35-532 in nonexperimental design studies and (2) heterogeneity of samples including patients from stroke, surgery or ICU units. These weaknesses may limit the generality of the findings. However, most studies used reliable and valid measurements and designed experimental studies, establishing causal relationships.

The process of reviewing the literature also has potential limitations. A publication bias may exist. The findings of this literature review were based on published literature and it is possible that important and relevant findings from key primary data articles were omitted as they were not included in the literature review. Moreover, a language bias may have been presented because we included only articles published in English-language journals. In addition, a variety of outcome measures (32 different ones in 36 studies) may limit the comparability of the findings.

\section{Conclusion}

Although immobility is known to cause functional decline or complications such as accelerated bone loss, muscle atrophy, malnutrition, delirium, sensory deprivation and incontinence (Creditor 1993, Convertino 1997, Markey \& Brown 2002, Timmerman 2007), nurses have not consistently ensured that their patients are ambulated (Kalisch et al. 2011). Covinsky et al. (2003) found that hospitalised older adults are often discharged from acute care hospitals with activities of daily living functioning that is worse than their baseline functioning. It may be that the importance of ambulation has been overlooked by nurses or patient mobilisation is not fully implemented by nurses. The findings of this literature review have provided insight into the impact of inpatient mobilisation (physical, psychological, social and organisational outcomes). Given the positive impact of mobilisation uncovered in this review, the mobilisation of inpatients should become a higher priority for nurses practicing in the inpatient setting and should not be neglected.

\section{Relevance to clinical practice}

The findings of this review suggest directions for further research. Interventions and policies that increase inpatient mobilisation need to be developed, tested and put into practice. Larger studies with a variety of populations employing blinded trial methodology or using predictive designs are needed. In addition to formulating interventions and policies to increase patient mobility, steps must be taken to ensure that the required work environment (e.g. adequate staffing, levels, teamwork and equipment) is provided to facilitate patient mobility. Studies exploring the reasons nurses miss nursing care include too few staff, poor use of existing staff resources, the time required for the nursing intervention, poor teamwork or communication problems, ineffective delegation, habit and denial (Kalisch 2006, Kalisch et al. 2011). The factors related to not mobilising patients need to be addressed by hospital organisations, nursing management and practicing clinical nurses.

\section{Acknowledgements}

No sources of support to disclose.

\section{Contributions}

Study design: BJK, SL, BWD; data collection and analysis: BJK, SL, BWD and manuscript preparation: BJK, SL, BWD. 


\section{References}

Augustin AC, de Quadros AS \& SarmentoLeite RE (2010) Early sheath removal and ambulation in patients submitted to percutaneous coronary intervention: a randomized clinical trial. International Journal of Nursing Studies 47, 939-945.

Behnke M, Jorres RA, Kirsten D \& Magnussen $\mathrm{H}$ (2003) Clinical benefits of a combined hospital and home-based exercise programme over 18 months in patients with severe COPD. Monaldi Archives for Chest Disease 59, 44-51.

Brown CJ, Friedkin RJ \& Inouye SK (2004) Prevalence and outcomes of low mobility in hospitalized older patients. Journal of the American Geriatrics Society 52, 1263-1270.

Browning L, Denehy L \& Scholes RL (2007) The quantity of early upright mobilisation performed following upper abdominal surgery is low: an observational study. The Australian Journal of Physiotherapy 53, 47-52.

Callen BL, Mahoney JE, Grieves CB, Wells TJ \& Enloe M (2004) Frequency of hallway ambulation by hospitalized older adults on medical units of an academic hospital. Geriatric Nursing 25, 212-217.

Chair SY, Thompson DR \& Li SK (2007) The effect of ambulation after cardiac catheterization on patient outcomes. Journal of Clinical Nursing 16, 212-214.

Chandrasekaran S, Ariaretnam SK, Tsung J \& Dickison D (2009) Early mobilization after total knee replacement reduces the incidence of deep venous thrombosis. ANZ Journal of Surgery 79, 526-529.

Chang PH, Lai YH, Shun SC, Lin LY, Chen ML, Yang Y, Tsai JC, Huang GS \& Cheng SY (2008) Effects of a walking intervention on fatigue-related experiences of hospitalized acute myelogenous leukemia patients undergoing chemotherapy: a randomized controlled trial. Journal of Pain and Symptom Management 35, 524-534.

Convertino VA (1997) Cardiovascular consequences of bed rest: effect on maximal oxygen uptake. Medicine \& Science in Sports \& Exercise 29, 191-196.

Covinsky KE, Palmer RM, Fortinsky RH, Counsell SR, Stewart AL, Kresevic D,
Burant CJ \& Landefeld CS (2003) Loss of independence in activities of daily living in older adults hospitalized with medical illness: increased vulnerability with age. Journal of the American Geriatrics Society 51, 451458.

Craig LE, Bernhardt J, Langhorne P \& Wu O (2010) Early mobilization after stroke: an example of an individual patient data meta-analysis of a complex intervention. Stroke 41, 2632-2636.

Creditor MC (1993) Hazards of hospitalization of the elderly. Annals of Internal Medicine 118, 219-223.

Cumming TB, Collier J, Thrift AG \& Bernhardt J (2008) The effect of very early mobilization after stroke on psychological well-being. Journal of Rehabilitation Medicine 40, 609-614.

Cumming TB, Thrift AG, Collier JM, Churilov L, Dewey HM, Donnan GA \& Bernhardt J (2011) Very early mobilization after stroke fast-tracks return to walking: further results from the phase II AVERT randomized controlled trial. Stroke 42, 153-158.

De Cordova PB, Phibbs CS, Bartel AP \& Stone PW (2012) Twenty-four/seven: a mixed-method systematic review of the off-shift literature. Journal of Advanced Nursing 68, 1454-1468.

Delaney CP, Zutshi M, Senagore AJ, Remzi FH, Hammel J \& Fazio VW (2003) Prospective, randomized, controlled trial between a pathway of controlled rehabilitation with early ambulation and diet and traditional postoperative care after laparotomy and intestinal resection. Diseases of the Colon of Rectum 46, 851-859.

Estrabrooks CA (2003) Individual determinants of research utilization: a systematic review. Journal of Advanced Nursing 43, 506-520.

Fisher SR, Kuo YF, Graham JE, Ottenbacher KJ \& Ostir GV (2010) Early ambulation and length of stay in older adults hospitalized for acute illness. Archives of Internal Medicine 170, 1942-1943.

Fisher SR, Galloway R, Kuo YF, Graham JE, Ottenbacher KJ, Ostir GV \& Goodwin JS (2011) Pilot study examining the association between ambulatory activity and falls among hospitalized older adults. Archives of Internal Medicine 92, 2090-2092.

Frenea S, Chirossel C, Rodriguez R, Baguet JP, Racinet C \& Payen JF (2004) The effects of prolonged ambulation on labor with epidural analgesia. Anesthesia \& Analgesia 98, 224-229.

García Guerrero JJ, Fernández de la Concha Castañeda J, López Quero D, Collado Bueno G, Infante de la Torre JR, Rayo Madrid JI \& Redondo Méndez A (2010) Lower incidence of venous thrombosis with temporary active-fixation lead implantation in mobile patients. Europace 12, 1604-1607.

Graf C (2006) Functional decline in hospitalized older adults. American Journal of Nursing 106, 58-78.

Hirsch C, Sommers L, Olsen A, Mullen L \& Winogard C (1990) The natural history of functional morbidity in hospitalized older patients. Journal of the American Geriatrics Society 38, 1296 1303.

Hirschhorn AD, Richards D, Mungavon SF, Morris NR \& Adams L (2008) Supervised moderate intensity exercise improves distance walked at hospital discharge following coronary artery bypass graft surgery - a randomised controlled trial. Heart, Lung \& Circulation 17, 129-138.

Indredavik B, Bakke F, Slordahl SA, Rokseth R \& Håheim LL (1999) Treatment in a combined acute and rehabilitation stroke unit: which aspects are most important? Stroke 30, 917-923.

Kalisch BJ (2006) Missed nursing care: a qualitative study. Journal of Nursing Care Quality 21, 306-313.

Kalisch BJ, Landstrom GL \& Hinshaw AS (2009a) Missed nursing care: a concept analysis. Journal of Advanced Nursing 65, 1509-1517.

Kalisch BJ, Landstrom G \& Williams RA (2009b) Missed nursing care: errors of omission. Nursing Outlook 57, 3-9.

Kalisch BJ, Tschannen D, Lee $\mathrm{H}$ \& Friese CR (2011) Hospital variation in missed nursing care. American Journal of Medical Quality 26, 291299.

Kamel HK, Iqbal MA, Mogallapu R, Maas D \& Hoffmann RG (2003) Time to ambulation after hip fracture surgery: relation to hospitalization outcomes. 
The Journals of Gerontology 58, 1042-1045.

Killey B \& Watt E (2006) The effect of extra walking on the mobility, independence and exercise self-efficacy of elderly hospital in-patients: a pilot study. Contemporary Nurse 22, 120-133.

Kurabe S, Ozawa T, Watanabe T \& Aiba $T$ (2010) Efficacy and safety of postoperative early mobilization for chronic subdural hematoma in elderly patients. Acta Neurochirurgica 52, 1171-1174.

Langhorne P, Stott D, Knight A, Bernhardt J, Barer D \& Watkins C (2010) Very early rehabilitation or intensive telemetry after stroke: a pilot randomised trial. Cerebrovascular Disease 29, 352-360.

Larsen K, Hansen TB, Thomsen PB, Christiansen T \& Soballe K (2009) Cost-effectiveness of accelerated perioperative care and rehabilitation after total hip and knee arthroplasty. The Journal of Bone \& Joint Surgery 91, 761-772.

Mahoney JE, Sager MA \& Jalaluddin M (1998) New walking dependence associated with hospitalization for acute medical illness: incidence and significance. The Journals of Gerontology. Series A, Biological Sciences and Medical Sciences 53, M307-M312.

Markey DW \& Brown RJ (2002) An interdisciplinary approach to addressing patient activity and mobility in the medical-surgical patient. Journal of Nursing Care Quality 16, 1-12.

Mundy LM, Leet TL, Darst K, Schnitzler MA \& Dunagan WC (2003) Early mobilization of patients hospitalized with community-acquired pneumonia. Chest 124, 883-889.

Miracapillo G, Costoli A, Addonisio L, Breschi M, Pasquinelli K, Gemignani L \& Severi S (2006) Early mobilization after pacemaker implantation. Journal of Cardiovascular Medicine 7, 197-202.
Nakao S, Takata S, Uemura H, Nakano S, Egawa H, Kawasaki Y, Kashihara M \& Yasui N (2010) Early ambulation after total knee arthroplasty prevents patients with osteoarthritis and rheumatoid arthritis from developing postoperative higher levels of D-dimer. The Journal of Medical Investigation 57, 146-151.

Oldmeadow LB, Edwards ER, Kimmel LA, Kipen E, Robertson VJ \& Bailey MJ (2006) No rest for the wounded: early ambulation after hip surgery accelerates recovery. ANZ Journal of Surgery 76, 607-611.

Padula CA, Hughes C \& Baumhover L (2009) Impact of nurse-driven mobility protocol on functional decline in hospitalized older adults. Journal of Nursing Care Quality 24, 325-331.

Partsch H \& Blattler W (2000) Compression and walking versus bed rest in the treatment of proximal deep venous thrombosis with low molecular weight heparin. Journal of Vascular Surgery 32, 861-869.

Pearse EO, Caldwell BF, Lockwood RJ \& Holland J (2007) Early mobilisation after conventional knee replacement may reduce the risk of postoperative venous thromboembolism. The Journal of Bone \& Joint Surgery 89, 316-322.

Rath S, Schreuders TAR, Stam HJ, Hovius SER \& Selles RW (2010) Early active motion versus immobilization after tendon transfer for foot drop deformity: a randomized clinical trial. Clinical Orthopaedics and Related Research 468, 2477-2484.

Rezaei-Adaryani M, Ahmadi F \& AsghariJafarabadi M (2009) The effect of changing position and early ambulation after cardiac catheterization on patients' outcomes: a single-blind randomized controlled trial. International Journal of Nursing Studies 46, 1047 1053.
Schweickert WD, Pohlman MC, Pohlman AS, Nigos C, Pawlik AJ, Esbrook CL, Spears L, Miller M, Franczyk M, Deprizio D, Schmidt GA, Bowman A, Barr R, McCallister KE, Hall JB \& Kress JP (2009) Early physical and occupational therapy in mechanically ventilated, critically ill patients: a randomised controlled trial. Lancet 373, 1874-1882.

Shadmi E \& Zisberg A (2011) In-hospital mobility and length of stay. Archives of Internal Medicine 171, 12981299.

Siu AL, Penrod JD, Boockvar KS, Koval K, Strauss E \& Morrison RS (2006) Early ambulation after hip fracture: effects on function and mortality. Archives of Internal Medicine 166, 766-771.

Tay-Teo K, Moodie M, Bernhardt J, Thrift AG, Collier J, Donnan G \& Dewey H (2008) Economic evaluation alongside a phase II, multi-centre, randomized controlled trial of very early rehabilitation after stroke (AVERT). Cerebrovascular Disease 26, 475-481.

Timmerman RA (2007) A mobility protocol for critically ill adults. Dimensions of Critical Care Nursing 26, 175-179.

Tyedin K, Cumming TB \& Bernhardt J (2010) Quality of life: an important outcome measure in a trial of very early mobilisation after stroke. Disability and Rehabilitation 32, 875-884.

Wong CA \& Cummings GG (2007) The relationship between nursing leadership and patient outcomes: a systematic review. Journal of Nursing Management 15, 508-521.

Zisberg A, Shadmi E, Sinoff G, Gur-Yaish N, Srulovici E \& Admi H (2011) Low mobility during hospitalization and functional decline in older adults. Journal of the American Geriatrics Society 59, 266-273. 
The Journal of Clinical Nursing (JCN) is an international, peer reviewed journal that aims to promote a high standard of clinically related scholarship which supports the practice and discipline of nursing.

For further information and full author guidelines, please visit JCN on the Wiley Online Library website: http:// wileyonlinelibrary.com/journal/jocn

\section{Reasons to submit your paper to JCN:}

High-impact forum: one of the world's most cited nursing journals, with an impact factor of 1.316 - ranked 21/101 (Nursing (Social Science)) and 25/103 Nursing (Science) in the 2012 Journal Citation Reports ${ }^{\circledR}$ (Thomson Reuters, 2012). One of the most read nursing journals in the world: over 1.9 million full text accesses in 2011 and accessible in over 8000 libraries worldwide (including over 3500 in developing countries with free or low cost access).

Early View: fully citable online publication ahead of inclusion in an issue.

Fast and easy online submission: online submission at http://mc.manuscriptcentral.com/jcnur.

Positive publishing experience: rapid double-blind peer review with constructive feedback.

Online Open: the option to make your article freely and openly accessible to non-subscribers upon publication in Wiley Online Library, as well as the option to deposit the article in your preferred archive. 